\title{
Impact of the H3K27M mutation on survival in pediatric high-grade glioma: a systematic review and meta-analysis
}

\author{
Victor M. Lu, MD, ${ }^{1}$ Mohammed A. Alvi, MBBS, ${ }^{2,3}$ Kerrie L. McDonald, PhD, ${ }^{1}$ and \\ David J. Daniels, MD, PhD²
}

'Prince of Wales Clinical School, The University of New South Wales, Sydney, Australia; and 2Department of Neurologic Surgery and ${ }^{3}$ Neuro-Informatics Laboratory, Mayo Clinic, Rochester, Minnesota

\begin{abstract}
OBJECTIVE Pediatric high-grade gliomas (pHGGs), including diffuse intrinsic pontine glioma, present a prognostic challenge given their lethality and rarity. A substitution mutation of lysine for methionine at position 27 in histone $\mathrm{H} 3$ (H3K27M) has been shown to be highly specific to these tumors. Data are accumulating regarding the poor outcomes of patients with these tumors; however, the quantification of pooled outcomes has yet to be done, which could assist in prioritizing management. The aim of this study was to quantitatively pool data in the current literature on the H3K27M mutation as an independent prognostic factor in $\mathrm{PHGG}$.
\end{abstract}

METHODS Searches of seven electronic databases from their inception to March 2018 were conducted according to the Preferred Reporting Items for Systematic Reviews and Meta-Analyses (PRISMA) guidelines. Data were extracted and pooled using a meta-analysis of proportions. Meta-regression was used to identify potential sources of heterogeneity.

RESULTS Six observational studies satisfied the selection criteria for inclusion. They reported the survival outcomes of a pooled cohort of 474 pHGG patients, with 258 (54\%) and $216(46 \%)$ patients positive and negative, respectively, for the H3K27M mutation. Overall, the presence of the mutation was independently and significantly associated with a worse prognosis (HR 3.630, $p<0.001$ ). Overall survival was significantly shorter (by 2.300 years; $p=0.008$ ) when the H3K27M mutation was present in PHGG. Meta-regression did not identify any study covariates of heterogeneous concern.

CONCLUSIONS According to the current literature, pHGG patients positive for the H3K27M mutation are more than 3 times more susceptible to succumbing to this disease by more than 2 years, compared to patients negative for the mutation. More robust outcome data are required to improve our quantitative understanding of this pathological entity in order to assist in prioritizing clinical management. Future larger prospective studies are required to overcome inherent biases in the current literature to validate the quantitative findings of this study.

https://thejns.org/doi/abs/10.3171/2018.9.PEDS18419

KEYWORDS H3K27M; histone 3; pediatric; high-grade glioma; glioblastoma; diffuse intrinsic pontine glioma; midline glioma; prognosis; oncology

$\mathrm{P}$ EDIATRIC high-grade gliomas (pHGGs), which incorporate WHO grades III and IV tumors, including diffuse intrinsic pontine glioma (now considered a diffuse midline glioma), are one of the leading causes of cancer-related morbidity and death in children. ${ }^{25,29}$ Overall survival (OS) is poor, with median estimates ranging from 4 to 17 months despite the best standard of care. ${ }^{17}$ Because of their lethality and eloquent locations, which often pre- clude biopsy and surgery, these tumors remain a difficult entity to study; thus, significant improvements in outlook have been lacking. ${ }^{45}$

The WHO combined both histological and molecular features for the first time in 2016, with one novel entry named "diffuse midline glioma, H3K27M mutant," a pHGG that typically arises in both children and young adults. ${ }^{25,26}$ This new set of classifications implicates the

ABBREVIATIONS CI = confidence interval; GRADE = Grades of Recommendation Assessment, Development and Evaluation; HR = hazard ratio; MD = mean difference; NOS = Newcastle-Ottawa Scale; OS = overall survival; pHGG = pediatric high-grade glioma; PRISMA = Preferred Reporting Items for Systematic Reviews and MetaAnalyses; RE = random effects.

SUBMITTED July 3, 2018. ACCEPTED September 6, 2018.

INCLUDE WHEN CITING Published online November 30, 2018; DOI: 10.3171/2018.9.PEDS18419. 
detection of $\mathrm{H} 3 \mathrm{~K} 27 \mathrm{M}$, which may prove clinically significant in $\mathrm{pHGG}$ when the mutation is detected since it is not exclusive to midline glioma. ${ }^{24}$ Biologically, the mutation can occur in several $\mathrm{H} 3$ genes including either $H 3 F 3 A$ or $H I S T I H 3 B / C$, which encode for histone 3 variants $\mathrm{H} 3.3$ and H3.1, respectively. ${ }^{24}$ There is a substitution of lysine (K) for methionine (M) at position 27 , which results in significant epigenetic reprogramming that has been shown to contribute to tumorigenesis. ${ }^{3,5,32,43}$ The K27 residue is, in fact, vulnerable to both methylation and acetylation changes, making these $\mathrm{H} 3$ variants subject to multiple possible posttranslational modifications. ${ }^{28,36}$ The H3K27M mutation itself drives the global loss of di- and trimethylation of histone H3K27 (H3K27me2 and H3K27me3) on wildtype histone proteins. ${ }^{2,5,22,43}$ It is this hypomethylation that has been shown to reprogram the epigenetic landscape to be more hospitable to tumorigenic change and, consequently, a poorer prognosis., ${ }^{2,4}$

Recently, Karremann et al. ${ }^{18}$ published a study in which they identified the H3K27M mutation as a universal poor indicator in pHGG at all locations of the central nervous system. Given the importance of this conclusion, there is great benefit to clinicians in quantifying this survival effect for $\mathrm{pHGG}$ in order to better prioritize clinical management of this rapid, dismal disease. In the context of how rapidly the clinical course of pHGG can deteriorate, clinicians could prioritize one management strategy over another based on available time in order to optimize the time/benefit ratio. To date, this quantification has not been done despite a number of individual studies on the H3K27M mutation as a prognostic factor in $\mathrm{PHGG}$ in the literature. ${ }^{3,4,18,19,21,37,44}$ The aim of the present systematic review and meta-analysis was to pool all currently published data on the H3K27M mutation in pHGG as a prognostic factor and to quantify its survival impact.

\section{Methods}

\section{Search Strategy}

The literature search strategy was designed around the PICOT question format: ${ }^{39}$ Do pHGG patients (Patient problem) with the H3K27M mutation, including both the H3.1 and H3.3 variant, (Intervention) compared to those without (Comparator), have a different prognosis of survival (Outcome) in observational cohort studies (Type of study)? Our review was conducted according to the Preferred Reporting Items for Systematic Reviews and MetaAnalyses (PRISMA) guidelines and recommendations. ${ }^{30}$ Electronic searches were performed using Ovid Embase, PubMed, Scopus, Cochrane Central Register of Controlled Trials, Cochrane Database of Systematic Reviews, American College of Physicians (ACP) Journal Club, and Database of Abstracts of Review of Effectiveness (DARE) from their dates of database inception to March 2018. The literature involving all comparative studies was searched using the following string of medical subject heading (MeSH) terms: ("glioma"[MeSH Terms] OR "glioma"[All Fields]) AND (H3K27 M[All Fields] OR K27 M[All Fields]). All identified articles were then systematically and independently assessed against the inclusion and exclusion criteria by two investigators (V.M.L. and M.A.A.).

\section{Selection Criteria}

The inclusion criteria used to screen all identified articles were as follows: 1) confirmed histopathological cases of pHGG; 2) a study cohort both with and without mutation at $\mathrm{H} 3$, either the $\mathrm{H} 3.1$ or $\mathrm{H} 3.3$ variant, involving the K27M substitution; 3) at least one primary outcome of either prognostic hazard ratio (HR) or OS mean difference (MD) accompanied by variance estimation; 4) an HR calculated from an adjusted Cox multivariate regression analysis if the primary outcome was HR; and 5) patient ages $\leq 18$ years. The exclusion criteria applied to all identified articles reporting outcomes: 1) patients with ages > 18 years, 2) data from univariate analyses if the HR was the primary outcome, and 3) involvement of low-grade glioma. All publications were limited to those involving human subjects and in the English language. Reviews, abstracts, case reports, conference presentations, editorials, and expert opinions were excluded to minimize potential publication bias and duplication of results.

\section{Data Extraction}

All data were extracted from article texts, tables, and figures with any estimates made based on the presented data and figures. The HR and MD were used as the summary statistics of this study. The primary outcome of interest was prognostic effect of the H3K27M mutation as inferred by an HR and its respective 95\% confidence interval (CI). The secondary outcome of absolute OS difference was inferred by an MD and its respective 95\% CI. Two reviewers (V.M.L. and M.A.A.) independently extracted data from article texts, tables, and figures, with any discrepancy resolved by discussion to reach consensus.

\section{Meta-Analysis}

Outcomes of the included studies were pooled together by meta-analysis, the HR statistic via logit transformation, to provide an overall summary statistic. This was presented as a forest plot. The $\mathrm{I}^{2}$ statistic was used to estimate the percentage of total variation across studies, owing to heterogeneity rather than chance, with values $>50 \%$ considered as substantial heterogeneity. ${ }^{15}$ Furthermore, the p-heterogeneity was assessed using the Cochran Q test. A random effects (RE) model was tested to take into account the possible clinical diversity and methodological variation between studies, as it assumes unequal variance between studies and distributes statistical weighting more conservatively. ${ }^{8}$ Meta-regression was performed to analyze potential effect modification by study publication year, mean age, study size, proportion of males, and H3 type (mixed vs H3.3 alone) on overall prognostic trend. All $\mathrm{p}$ values were 2 -sided with significance set at $\mathrm{p}<$ 0.05. Statistical analyses were conducted with Stata 14.1 (StataCorp).

\section{Bias and Quality Assessment}

Bias in publication was assessed through the generation of funnel plots. Small-study bias was assessed using Egger's test and validated with Begg's test. ${ }^{40}$ A trim-and-fill method was used to recalculate pooled effect size if significant bias was still suspected.9 The final inclusion of any 


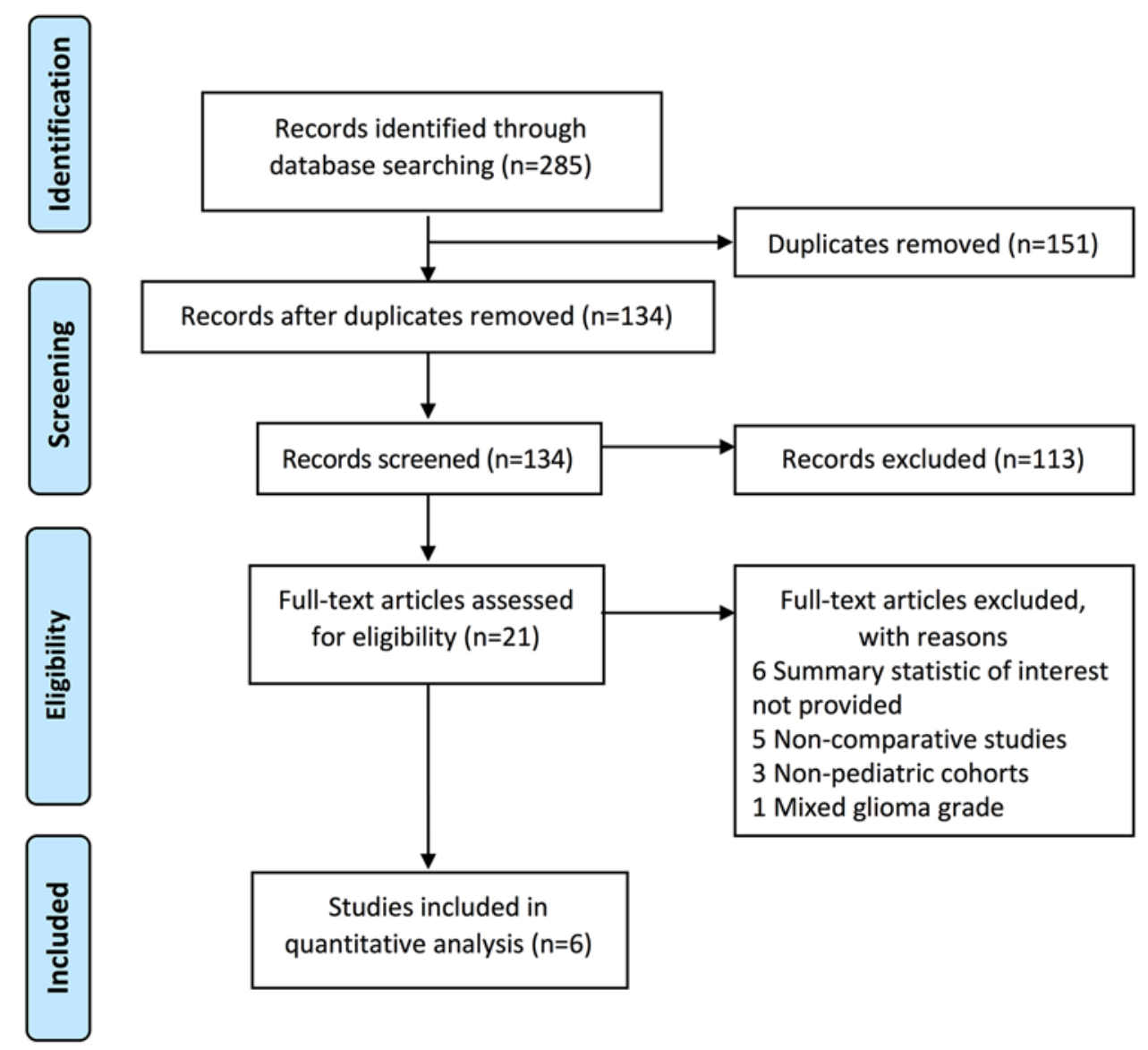

FIG. 1. Results of the search strategy as performed by under the PRISMA guidelines. Figure is available in color online only.

outlying study was reconsidered in the context of overall trend direction and significance upon their exclusion. The strength of evidence for each outcome was evaluated using the Grades of Recommendation Assessment, Development and Evaluation (GRADE) approach and presented as a summary-of-findings table to identify the certainty of all pooled outcomes. ${ }^{1}$ The GRADE system assesses the quality of a pooled outcome (from high to very low) based on type, design, consistency, and generalizability of each contributing study, indicating how likely further research will affect the estimate of effect calculated by this meta-analysis. ${ }^{13}$ Each study was then assessed against the Newcastle-Ottawa Scale (NOS), ${ }^{7,46}$ which accounts for selection, comparability, and outcome to evaluate the quality of nonrandomized studies. The NOS score serves as a surrogate indication for risk of inherent bias in the incorporated data of each study.

\section{Results}

\section{Literature Search}

The search strategy identified a total of 285 studies (Fig. 1). After the removal of 151 duplicate studies, inclusion and exclusion criteria were applied to titles and abstracts of the remaining 134 articles. This yielded 21 studies that underwent full-text analysis. Six retrospective observa- tional studies $3,4,18,19,21,44$ satisfied all criteria for inclusion into the meta-analysis. All studies were determined to be of good quality according to the NOS criteria ${ }^{46}$ (median score 8; Supplementary Data), and their design characteristics are summarized in Table 1.

\section{Demographics and Clinical Features}

Overall, there were $474 \mathrm{pHGG}$ patients incorporated into the relevant analyses across all included studies (Table 1). Pooled values for median age at diagnosis and male proportion were 9.17 years and $51 \%$, respectively. There were $258(54 \%)$ and $216(46 \%)$ cases in the H3K27M and non-H3K27M cohorts, respectively. Sanger sequencing was the only technique used to determine mutation status in four studies, ${ }^{4,19,21,44}$ both Sanger and next-generation sequencing was used in one study, ${ }^{3}$ and immunohistochemistry and pyrosequencing were used in the other. ${ }^{18}$

In terms of clinical features, three studies ${ }^{3,19,44}$ reported outcomes for the H3K27M mutation of the H3.3 variant only, with the other three reporting outcomes for mixed H3.1 and H3.3 cohorts (Table 2). Tumor locations throughout the central nervous system were reported, including in the cortex, basal ganglia, brainstem, and spinal cord. Three studies ${ }^{3,419}$ reported outcomes for the brainstem only, with biopsy the only surgical intervention. In the other studies, resection was also utilized. Finally, radiation therapy and 


\begin{tabular}{|c|c|c|c|c|c|c|c|c|c|c|}
\hline \multirow[b]{3}{*}{ Authors \& Year } & \multirow[b]{3}{*}{ Country } & \multirow[b]{3}{*}{ Design* } & \multirow[b]{3}{*}{$\begin{array}{c}\text { Study } \\
\text { Quality† }\end{array}$} & \multirow[b]{3}{*}{$\begin{array}{l}\text { Study } \\
\text { Period }\end{array}$} & \multicolumn{3}{|c|}{ Overall } & & & \multirow[b]{3}{*}{ Detection Method } \\
\hline & & & & & \multirow{2}{*}{$\begin{array}{l}\text { No. } \\
\text { of } \\
\text { Pts }\end{array}$} & \multirow{2}{*}{$\begin{array}{c}\text { Median } \\
\text { Age at } \\
\text { Dx (yrs) }\end{array}$} & \multirow[b]{2}{*}{$\begin{array}{c}\% \\
\text { Males }\end{array}$} & \multicolumn{2}{|c|}{ Cohort (no. [\% overall]) } & \\
\hline & & & & & & & & $\begin{array}{l}\text { H3K27M } \\
\text { Cohort }\end{array}$ & $\begin{array}{c}\text { Non-H3K27M } \\
\text { Cohort }\end{array}$ & \\
\hline $\begin{array}{l}\text { Buczkowicz et al., } \\
2014\end{array}$ & Canada & $\mathrm{R}, \mathrm{OCS}(1)$ & Good (8) & 1984-2012 & 66 & 6.47 & $51 \%$ & $42(64 \%)$ & $24(36 \%)$ & $\begin{array}{l}\text { Sanger, next-genera- } \\
\text { tion sequencing }\end{array}$ \\
\hline Castel et al., 2015 & France & $\mathrm{R}, \mathrm{OCS}(1)$ & Good (8) & NR & 89 & 7.76 & $51 \%$ & $78(88 \%)$ & $11(12 \%)$ & Sanger sequencing \\
\hline $\begin{array}{l}\text { Karremann et al., } \\
2018\end{array}$ & Germany & $\begin{array}{l}\mathrm{R}, \mathrm{OCS} \\
(\mathrm{NR})\end{array}$ & Good (9) & NR & 77 & 10.4 & $51 \%$ & $61(79 \%)$ & $16(21 \%)$ & $\mathrm{IHC}$, pyrosequencing \\
\hline $\begin{array}{l}\text { Khuong-Quang et al., } \\
2012\end{array}$ & $\begin{array}{l}\text { Canada, } \\
\text { Germany }\end{array}$ & $\mathrm{R}, \mathrm{OCS}(3)$ & Good (9) & NR & 42 & 7.12 & $50 \%$ & $30(71 \%)$ & $12(29 \%)$ & Sanger sequencing \\
\hline Korshunov et al., 2015 & Russia & $\mathrm{R}, \mathrm{OCS}(1)$ & Good (8) & 1998-2013 & 162 & 11 & $50 \%$ & $35(22 \%)$ & $127(78 \%)$ & Sanger sequencing \\
\hline Venneti et al., 2014 & US & $\mathrm{R}, \mathrm{OCS}(3)$ & Good (8) & NR & 38 & NR & NR & $12(32 \%)$ & $26(68 \%)$ & Sanger sequencing \\
\hline Pooled & & & & & 474 & 9.17 & $51 \%$ & $258(54 \%)$ & $216(46 \%)$ & \\
\hline
\end{tabular}

$\mathrm{Dx}=$ diagnosis; $\mathrm{IHC}$ = immunohistochemistry; $\mathrm{NR}=$ not reported; $\mathrm{OCS}=$ observational cohort study; pts = patients; $\mathrm{R}=$ retrospective.

* Number of institutions in parentheses.

$\dagger$ NOS score appears in parentheses.

chemotherapy were both used as adjuvant therapies in all four studies reporting such data.

\section{Prognostic Effect of H3K27M}

Detection of the H3K27M mutation in pHGG was significantly and independently associated with a worse prognosis. Across the five studies reporting multivariate-derived HRs (Table 3), there was a pooled HR of $3.630\left(95 \%\right.$ CI $\left.2.539-5.190, \mathrm{p}<0.001, \mathrm{I}^{2}=0.0 \%\right)$ for the $\mathrm{H} 3 \mathrm{~K} 27 \mathrm{M}$ mutation in $\mathrm{pHGG}$ derived by an RE model (Fig. 2).

Meta-regression revealed that differences in study year $(p=0.763)$, mean age $(p=0.834)$, study size $(p=0.419)$, proportion of males $(\mathrm{p}=0.867)$, and $\mathrm{H} 3$ type (both H3.1 and H3.3 [reference] vs H3.3 alone, $p=0.549$ ) between the included studies did not significantly affect the pooled result.

\section{Overall Survival MD}

The OS of patients with pHGG was significantly less when H3K27M was detected. Across the four studies reporting OS, the pooled MD in OS was 2.300 years shorter (95\% CI $0.611-3.989$ years; $p=0.008 ; I^{2}=97.0 \%$ ) in $\mathrm{pHGG}$ with the H3K27M mutation derived by RE model (Fig. 3).
Pooled OS values in the H3K27M and non-H3K27M cohorts were 0.91 and 4.35 years, respectively.

Meta-regression revealed that differences in study year $(p=0.637)$, mean age $(p=0.922)$, study size $(p=0.463)$, proportion of males ( $p=0.867$ ), and H3 type (both H3.1 and H3.3 [reference] vs H3.3 alone, $p=0.850$ ) between the included studies did not significantly affect the pooled result.

\section{Bias and Study Quality}

For the HR summary statistic, the funnel plot generated to assess for overall publication bias did not appear asymmetrical (Supplementary Data), and small-study bias was not implicated by Egger's $(\mathrm{p}=0.456)$ and Begg's ( $\mathrm{p}$ $=0.221$ ) test results. For the MD summary statistic, asymmetry was suspected in the funnel plot generated to assess for overall publication bias (Supplementary Data). Although small-study bias was not implicated by Egger's ( $p$ $=0.784)$ and Begg's $(p=0.734)$ test results, we performed a trim-and-fill test to account for our suspicion, which resulted in 1 trim-and-fill study added to the pooled statistic (Supplementary Data); however, both direction and significance of trend remained unchanged: MD 2.034 years shorter (95\% CI 0.465-3.603 years; $\mathrm{p}=0.011$ ).

TABLE 2. Clinical features of included studies

\begin{tabular}{lllll}
\hline \multicolumn{1}{c}{ Authors \& Year } & H3 Variant(s) Tested & Tumor Location & Surgical Intervention & Adjuvant Mgmt \\
\hline Buczkowicz et al., 2014 & H3.3 & Brainstem & Biopsy & Biopsy \\
\hline Castel et al., 2015 & H3.1, H3.3 & Brainstem & Resection, biopsy & RT \\
\hline Karremann et al., 2018 & H3.1, H3.3 & Cortex, basal ganglia, brainstem, spinal cord & Biopsy \\
\hline Khuong-Quang et al., 2012 & H3.3 & Brainstem & Resection, biopsy & RT, CT \\
\hline Korshunov et al., 2015 & H3.1, H3.3 & Cortex, basal ganglia, brainstem & Resection, biopsy & NR \\
\hline Venneti et al., 2014 & H3.3 & Cortex, basal ganglia, brainstem, spinal cord & RT \\
\hline
\end{tabular}

$\mathrm{CT}=$ chemotherapy $; \mathrm{Mgmt}=$ management $\mathrm{RT}=$ radiation therapy . 
Lu et al.

TABLE 3. Extracted outcomes of studies included in the meta-analysis

\begin{tabular}{lcccccc}
\hline & \multicolumn{3}{c}{ Multivariate Analysis of H3K27M } & \multicolumn{2}{c}{ OS (yrs) } \\
\cline { 2 - 6 } \multicolumn{1}{c}{ Authors \& Year } & $\mathrm{HR}$ & $95 \% \mathrm{Cl}$ & $\mathrm{p}$ Value & Other Covariates Included & $\mathrm{H} 3 \mathrm{~K} 27 \mathrm{M}$ & $\mathrm{Non}-\mathrm{H} 3 \mathrm{~K} 27 \mathrm{M}$ \\
\hline Buczkowicz et al., 2014 & 2.793 & $1.350-5.777$ & 0.006 & Age, histological diagnosis, sex & $0.63 \pm 1.77$ & $1.84 \pm 1.77$ \\
\hline Castel et al., 2015 & 5.150 & $1.954-6.890$ & $<0.001$ & Treatment type, imaging contrast enhancement & NR & NR \\
\hline Karremann et al., 2018 & $\mathrm{NR}$ & $\mathrm{NR}$ & $\mathrm{NR}$ & $\mathrm{NR}$ & $0.87 \pm 0.25$ & $4.54 \pm 0.82$ \\
\hline Khuong-Quang et al., 2012 & 4.277 & $1.264-14.472$ & 0.019 & Age, histological diagnosis & $0.73 \pm 0.48$ & $4.59 \pm 5.55$ \\
\hline Korshunov et al., 2015 & 2.84 & $1.38-5.87$ & 0.005 & Sex, location, amplification, alternative mutations & $1.04 \pm 0.40$ & $2.22 \pm 1.46$ \\
\hline Venneti et al., 2014 & 3.514 & $1.17-10.559$ & 0.025 & Age, histological diagnosis, location, sex & NR & NR \\
\hline
\end{tabular}

Values expressed as the mean \pm standard deviation, unless indicated otherwise.

The certainty of effect estimates for both HR and MD were deemed to be moderate and very low, respectively, per the GRADE criteria (Table 4). According to the NOS criteria, all studies were identified to be of good quality (Supplementary Data).

\section{Discussion}

In this systematic review and meta-analysis, we investigated the survival impact of the H3K27M mutation in pHGG. The pooled data revealed that the mutation indeed conferred an independent, worse prognosis in $\mathrm{pHGG}$ that is more than 3 times more severe than if the mutation were absent (HR 3.630, p < 0.001). We were able to quantify that OS was significantly shorter by more than 2 years in the presence of the H3K27M mutation (MD 2.300 years, $p$ $=0.008)$. However, clinical heterogeneity between studies remains a legitimate concern given the limited data currently available, and the OS difference is subject to biases in the current literature. Thus, although the trend toward a poorer prognosis in $\mathrm{pHGG}$ when the $\mathrm{H} 3 \mathrm{~K} 27 \mathrm{M}$ mutation is present is consistently reported in the literature, the degree to which this quantitatively prognosticates disease severity-and thus can help to prioritize management options-requires further investigation.

The worse prognosis conveyed by the H3K27M mutation in pHGG was consistently reported as significant and independent of other potential prognostic factors by each included study. Factors commonly associated with prognosis in adult glioma, such as age, sex, histology, location, and treatment regimens, have been widely reported as not significant in pHGG. $3,18,19,21,43$ Indeed, the reported locations across all areas of the central nervous system in the included studies seemed to imply that the poor prognosis of H3K27M is universal. ${ }^{18}$ Thus, it is even more important to understand the quantity by which the H3K27M mutation affects survival in pHGG. Because pHGG follows a rapid clinical course, the time available to execute effective intervention(s) is limited. ${ }^{42}$ If we can better un-

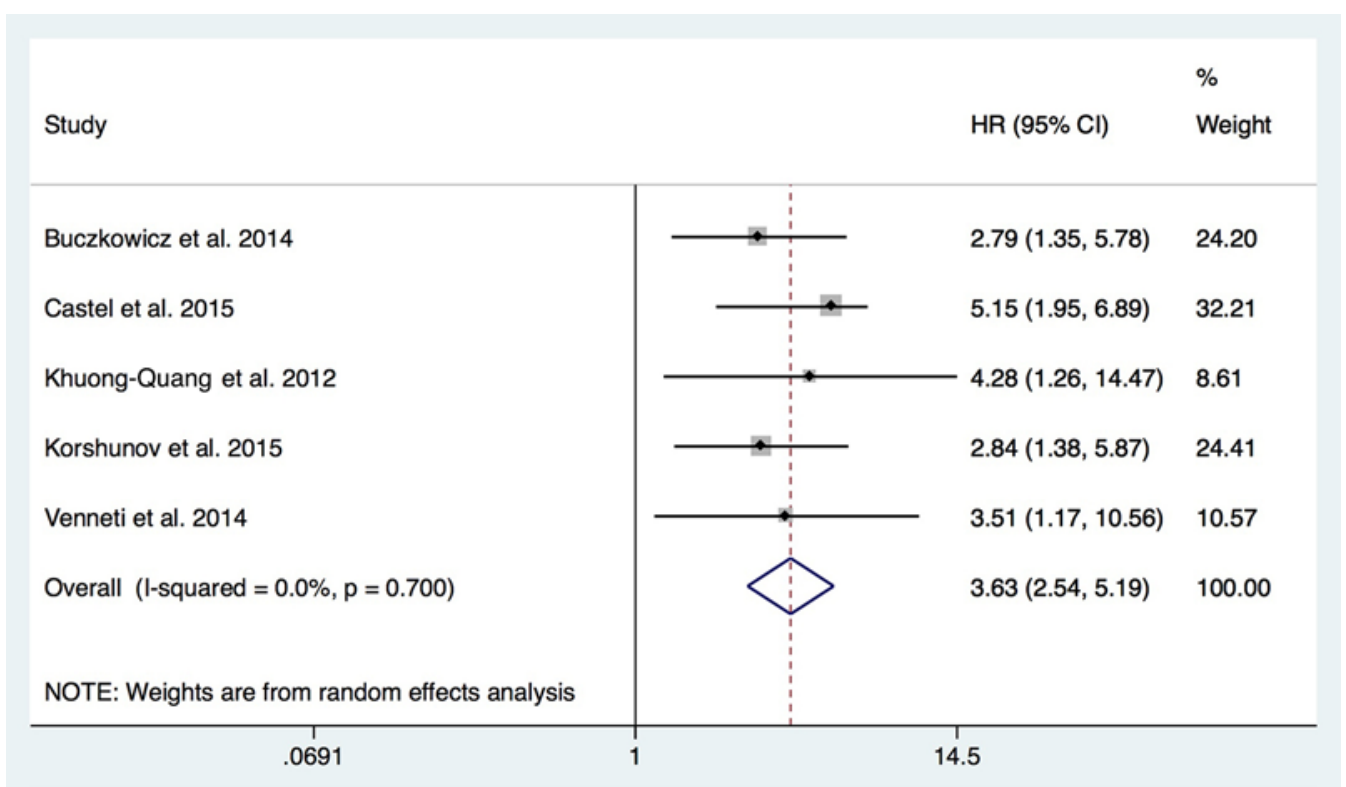

FIG. 2. A forest plot of the pooled HRs and their corresponding $95 \% \mathrm{Cls}$ for all studies investigating the presence of the H3K27M mutation in pHGG cohorts. The weighted $\mathrm{HR}, 95 \% \mathrm{Cl}$, and relative weightings are represented by the middle of the square, the horizontal line, and the relative size of the square, respectively. Figure is available in color online only. 


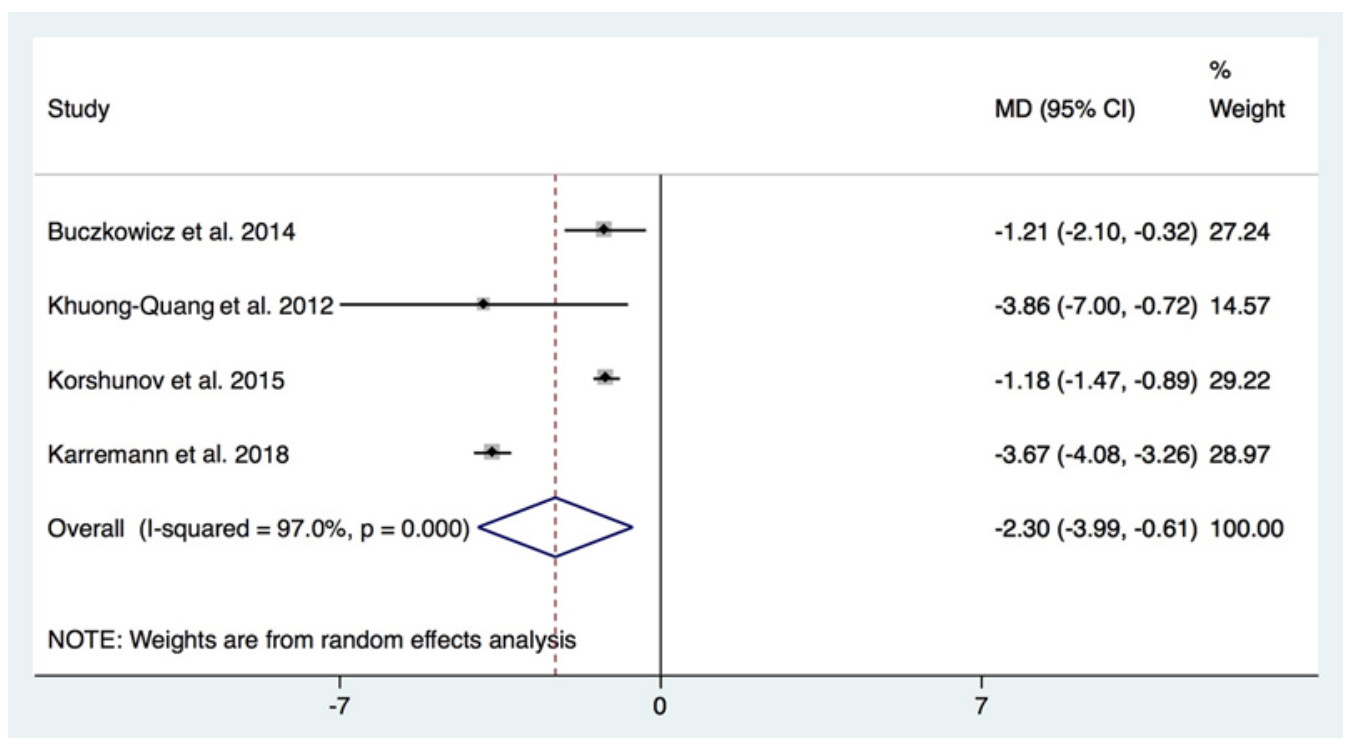

FIG. 3. A forest plot of pooled OS as by reported the MD of cohorts in studies investigating the presence of the H3K27M mutation in pHGG. The weighted MD, $95 \% \mathrm{Cl}$, and relative weightings are represented by the middle of the square, the horizontal line, and the relative size of the square, respectively. The overall statistics provided refer to overall heterogeneity. Figure is available in color online only.

derstand the quantity by which this mutation shortens OS, clinicians will be better informed about which options are most reasonable with respect to a time/benefit ratio, e.g., short-course versus long-course radiotherapy, and the urgency for chemotherapy intervention. ${ }^{10,14}$

It is very interesting that the prognostic effect exerted by this mutation appears consistent in trend and significance regardless of which of the two $\mathrm{H} 3$ variants is considered. Meta-regression for variant type (H3.3 vs H3.3 and H3.1) did not yield a significant modifying effect on OS; however, this analysis was limited given the absence of any studies reporting H3.1 outcomes only. Unfortunately, a paucity of literature directly compares the two variants clinically. Moving forward, it will be crucial to delineate the clinical course of the two. Although H3.1 and H3.3 differ in sequence by only five amino acids, these two $\mathrm{H} 3$ variants are quite distinct in terms of cellular function, including expression during the cell cycle, localization on the genome, and accumulation in pericentric moieties. ${ }^{11,41}$ These differences appear to have significant clinical consequences. In a study of pontine glioma, MacKay et al. ${ }^{27}$ indicated that the H3.1 variant is associated with significantly greater OS than the H3.3 variant. Castel et al. ${ }^{4}$ found that in pediatric midline glioma, cases with the H3K27M mutation in H3.3 were less responsive to radiation therapy, relapsed earlier, and exhibited more metastatic recurrences than the cases with the H3K27M mutation in H3.1. Furthermore, they also proposed that the $\mathrm{H} 3.1$ and $\mathrm{H} 3.3$ variants correspond to mesenchymal and proneural phenotypes, respectively, which have different and vastly significant ramifications in rendering a prognosis for pHGG and thus prioritizing clinical management. ${ }^{35}$

Ultimately, understanding how the H3K27M mutation promotes pHGG tumorigenesis can encourage diagnosis and the discovery of novel targets. This is extremely im- portant because there is currently no established standard of care for pHGG, particularly those located in the brainstem. ${ }^{31}$ From a therapeutic perspective, the hypomethylation associated with the $\mathrm{H} 3 \mathrm{~K} 27 \mathrm{M}$ mutation suggests a correlation between chromatin dysregulation and malignancy in pHGG. ${ }^{3,32,43}$ Novel agents that target the processes involved in epigenetic regulation are currently under investigation, including histone deacetylase (HDAC) inhibitors and BET bromodomain inhibitors, and may prove therapeutically advantageous in the future. ${ }^{12,34}$

Finally, although biopsy is gaining traction as an additional method of diagnosing this mutation, one particular hurdle with this intervention is the feasibility of obtaining tissue. In the future, a "liquid biopsy" may be a more practical alternative to confirm H3K27M status in pHGG patients, with indications that tumor-derived cell-free DNA (cfDNA) for H3K27M can be found in cerebrospinal fluid. ${ }^{16,38}$ Furthermore, detecting biomarkers for this specific mutation in the serum remains a plausible possibility in the future given that there is emerging evidence that the presence of pediatric glioma can already be detected. ${ }^{23}$

\section{Strengths and Limitations of the Current Literature}

This systematic review and meta-analysis adhered strictly to the PRISMA guidelines and its selection criteria. In order to improve the validity of the findings, the prognostic HRs were included only if they were derived from multivariate regression analysis. This was done to ensure that other potential prognostic factors would not confound the true impact of the H3K27M mutation in pHGG, as they were included as regression covariates. Moreover, cohorts were restricted to those representing HGG only. This was done to ensure that the better prognosis associated with low-grade glioma did not confound the prognostic effect of this mutation in HGG. Finally, while this mutation 
TABLE 4. GRADE assessment of primary outcomes to assess the level of evidence contributing to each outcome

\begin{tabular}{|c|c|c|c|c|c|c|c|c|c|c|}
\hline \multirow[b]{2}{*}{ Outcome } & \multirow[b]{2}{*}{$\begin{array}{l}\text { Summ } \\
\text { Stat }\end{array}$} & \multirow[b]{2}{*}{$\begin{array}{l}\text { Pooled Statistic } \\
\qquad(95 \% \mathrm{Cl})\end{array}$} & \multirow[b]{2}{*}{$\begin{array}{l}\text { No. of } \\
\text { Studies }\end{array}$} & \multicolumn{5}{|c|}{ Certainty Assessment } & \multirow[b]{2}{*}{$\begin{array}{c}\text { Overall } \\
\text { Quality Score }\end{array}$} & \multirow[b]{2}{*}{ Certainty } \\
\hline & & & & $\begin{array}{l}\text { Type of } \\
\text { Evidence }\end{array}$ & $\begin{array}{l}\text { Study } \\
\text { Quality }\end{array}$ & Consistency & Directness & $\begin{array}{l}\text { Effect } \\
\text { Size }\end{array}$ & & \\
\hline OS (primary) & $\mathrm{HR}$ & $3.630(2.539-5.190)$ & 5 & +2 & 0 & +1 & -1 & +1 & +3 & Moderate \\
\hline OS (secondary) & MD & $2.300(0.611-3.989)$ & 4 & +2 & -1 & +1 & -1 & 0 & +1 & Very low \\
\hline
\end{tabular}

Summ Stat = summary statistic.

The overall quality score is determined based on the sum of the included domains. Type of evidence is based on the design of the included studies (range +2 to +4 ). The study quality reflects the blinding and allocation, follow-up and withdrawals, sparsity of data, and methodological concerns (range -3 to 0 ). Consistency is graded based on the heterogeneity of included population and study end points with respect to one another (range -1 to +1 ). Directness is graded based on the generalizability of included results (range -2 to 0 ). Effect size is graded based on the magnitude of the pooled statistic being consistent and relation to score of 2 relative units (range 0 to +2 ). The overall quality of results for each outcome can be considered high ( $\geq 4$ points), moderate ( 3 points), low ( 2 points), or very low ( $\leq 1$ point).

is also found in adult glioma, it was important to restrict this study to pediatric ( $\leq 18$ years) cases, as the mutation's significance in adult glioma is less well understood. ${ }^{6}$ Specifically, the global genomic aberrations between adults and children are significantly different, making them biologically distinct, which has the potential to confound the clinical value of this mutation in pHGG only. ${ }^{20,33}$

The current literature is limited by a number of factors. The rare and lethal nature of pHGG has resulted in small cohorts to date. This makes assessment of interstudy bias difficult, as statistical analyses can be underpowered with these low numbers. ${ }^{40}$ Despite the good quality of the included studies and our efforts to minimize the impact of statistical heterogeneity by utilizing the RE model, the inherent degree of variation in clinical parameters could not be completely accounted for in this meta-analysis, including tumor burden at presentation, tumor location, adjuvant therapies, access to diagnostic services at all centers, accurate diagnoses, and family preference. This heterogeneity undoubtedly exerts a confounding impact on the overall result when studied retrospectively. Given that techniques other than gold-standard Sanger sequencing were used to determine mutation status, it is possible that the pooled results underestimate the true potency of this mutation currently. We note that included studies incorporated a wide range of clinical variables in their respective multivariate regression analyses, which will have reduced this potential impact somewhat. Additionally, meta-regression assists in identifying obvious covariates of modifying effect, none of which were found. However, better interstudy consistency and transparency in the clinical process will benefit the validity of pooling the literature in the future.

The pooling of data in our meta-analysis required data to describe the appropriately derived statistic (i.e., HR or $\mathrm{MD})$ and to be accompanied by its variance, which is not consistently reported or performed in the literature. This requirement excluded the cohort study of Karremann et al..$^{18}$ and the extensive meta-analysis of genomic data by Mackay et al., ${ }^{27}$ neither of which provided a quantification for the significantly worse prognosis each reported the H3K27M mutation confers in this population. Thus, while other survival data are published in the literature, their methodological design dictates their exclusion, and this limits the generalizability of our results. Additionally, the vast time period from which all cases have been collected in the studies may, in fact, overestimate the severity of the
H3K27M mutation given the greater number of adjuvant, targeted, and experimental therapies available today that dwarf those of prior decades. This is relevant because publication bias remains an important consideration in the current literature as well and given that our trim-and-fill analysis of the MD in OS outcome demonstrated a slightly smaller statistic than that in the reporting studies alone.

Surgical specimens are required to make the histopathological diagnosis of pHGG, as well as determine H3K27M status. However, as the role of biopsy in pHGG remains controversial, not all centers perform it, and this tempers our expectation of how representative the current literature is of this disease and mutation. In particular, it leaves current data vulnerable to two biases. The first is selection bias, for inclusion into a study of the H3K27M mutation requires biological diagnosis. Those who are unable to access diagnostic services, who are misdiagnosed, or who are managed without histopathological diagnosis will have been excluded from the included studies, with an unknown impact on our presented results. Given the great heterogeneity in our MD statistic $\left(\mathrm{I}^{2}=97 \%\right)$, it may be more appropriate to apply our findings to pHGG patients in whom the H3K27M mutation status is assessed, excluding those in whom a diagnosis is not made or tested for. This would consolidate the implications of the MD of 2.300 years in the context of expected pHGG survival between 4 and 17 months in general.

Second, the tendency to evaluate for this mutation prior to its incorporation into the WHO classification in 2016 is unknown and may have only been done and reported by centers with a vested interest in this particular field. Thus, a protopathic bias toward earlier intervention and management and a publication bias toward a significant effect in these studies cannot be currently discounted. Hence, how representative-and consequently how generalizable-the quantitative data from these studies are remains unclear. Future studies that are conducted in a prospective manner will be better equipped to validate the numerical findings of our study.

\section{Conclusions}

The H3K27M mutation is commonly found in pHGG. This study confirmed that the mutation confers a poor survival impact, with patients positive for the mutation more than 3 times more susceptible to succumbing to this disease 
by more than 2 years, compared to patients negative for the mutation. We propose the incorporation of tissue evaluation into the standard of care for pHGG when deemed safe, and we encourage further development of techniques to perform such assessments. Collectively, the correlation between molecular/genetic data, histopathology, and clinical outcomes will improve both the prioritization of available management options and the information provided to the patient and their family. Although the current literature is in agreement regarding the poor prognosis conveyed by the H3K27M mutation, it is small, and given the historic novelty of this mutation, the literature remains prone to influence by a number of bias concerns. Thus, the certainty of the current quantitative data is limited. This will be improved in the future by larger prospective studies to better inform clinicians on how to prioritize management based on the specific prognostic severity of the H3K27M mutation in $\mathrm{pHGG}$.

\section{References}

1. Atkins D, Best D, Briss PA, Eccles M, Falck-Ytter Y, Flottorp $\mathrm{S}$, et al: Grading quality of evidence and strength of recommendations. BMJ 328: 1490, 2004

2. Bender S, Tang Y, Lindroth AM, Hovestadt V, Jones DT, Kool M, et al: Reduced H3K27me3 and DNA hypomethylation are major drivers of gene expression in K27M mutant pediatric high-grade gliomas. Cancer Cell 24:660-672, 2013

3. Buczkowicz P, Bartels U, Bouffet E, Becher O, Hawkins C: Histopathological spectrum of paediatric diffuse intrinsic pontine glioma: diagnostic and therapeutic implications. Acta Neuropathol 128:573-581, 2014

4. Castel D, Philippe C, Calmon R, Le Dret L, Truffaux N, Boddaert N, et al: Histone H3F3A and HIST1H3B K27M mutations define two subgroups of diffuse intrinsic pontine gliomas with different prognosis and phenotypes. Acta Neuropathol 130:815-827, 2015

5. Chan KM, Fang D, Gan H, Hashizume R, Yu C, Schroeder M, et al: The histone H3.3K27M mutation in pediatric glioma reprograms H3K27 methylation and gene expression. Genes Dev 27:985-990, 2013

6. Daoud EV, Rajaram V, Cai C, Oberle RJ, Martin GR, Raisanen JM, et al: Adult brainstem gliomas with H3K27M mutation: radiology, pathology, and prognosis. J Neuropathol Exp Neurol 77:302-311, 2018

7. Deeks JJ, Dinnes J, D’Amico R, Sowden AJ, Sakarovitch C, Song F, et al: Evaluating non-randomised intervention studies. Health Technol Assess 7:iii-x, 1-173, 2003

8. DerSimonian R, Laird N: Meta-analysis in clinical trials. Control Clin Trials 7:177-188, 1986

9. Duval S, Tweedie R: Trim and fill: a simple funnel-plot-based method of testing and adjusting for publication bias in metaanalysis. Biometrics 56:455-463, 2000

10. Friedman GK, Spiller SE, Harrison DK, Fiveash JB, Reddy AT: Treatment of children with glioblastoma with conformal radiation, temozolomide, and bevacizumab as adjuncts to surgical resection. J Pediatr Hematol Oncol 35:e123-e126, 2013

11. Goldberg AD, Banaszynski LA, Noh KM, Lewis PW, Elsaesser SJ, Stadler S, et al: Distinct factors control histone variant H3.3 localization at specific genomic regions. Cell 140:678-691, 2010

12. Grasso CS, Tang Y, Truffaux N, Berlow NE, Liu L, Debily MA, et al: Functionally defined therapeutic targets in diffuse intrinsic pontine glioma. Nat Med 21:555-559, 2015

13. Guyatt GH, Oxman AD, Vist GE, Kunz R, Falck-Ytter Y,
Alonso-Coello P, et al: GRADE: an emerging consensus on rating quality of evidence and strength of recommendations. BMJ 336:924-926, 2008

14. Hankinson TC, Patibandla MR, Green A, Hemenway M, Foreman N, Handler M, et al: Hypofractionated radiotherapy for children with diffuse intrinsic pontine gliomas. Pediatr Blood Cancer 63:716-718, 2016

15. Higgins JPT, Thompson SG, Deeks JJ, Altman DG: Measuring inconsistency in meta-analyses. BMJ 327:557-560, 2003

16. Huang TY, Piunti A, Lulla RR, Qi J, Horbinski CM, Tomita T, et al: Detection of histone H3 mutations in cerebrospinal fluid-derived tumor DNA from children with diffuse midline glioma. Acta Neuropathol Commun 5:28, 2017

17. Jansen MH, van Vuurden DG, Vandertop WP, Kaspers GJ: Diffuse intrinsic pontine gliomas: a systematic update on clinical trials and biology. Cancer Treat Rev 38:27-35, 2012

18. Karremann M, Gielen GH, Hoffmann M, Wiese M, Colditz N, Warmuth-Metz M, et al: Diffuse high-grade gliomas with H3 K27M mutations carry a dismal prognosis independent of tumor location. Neuro Oncol 20:123-131, 2018

19. Khuong-Quang DA, Buczkowicz P, Rakopoulos P, Liu XY, Fontebasso AM, Bouffet E, et al: K27M mutation in histone H3.3 defines clinically and biologically distinct subgroups of pediatric diffuse intrinsic pontine gliomas. Acta Neuropathol 124:439-447, 2012

20. Kleinschmidt-DeMasters BK, Mulcahy Levy JM: H3 K27Mmutant gliomas in adults vs. children share similar histological features and adverse prognosis. Clin Neuropathol 37:53-63, 2018

21. Korshunov A, Ryzhova M, Hovestadt V, Bender S, Sturm D, Capper D, et al: Integrated analysis of pediatric glioblastoma reveals a subset of biologically favorable tumors with associated molecular prognostic markers. Acta Neuropathol 129:669-678, 2015

22. Lewis PW, Müller MM, Koletsky MS, Cordero F, Lin S, Banaszynski LA, et al: Inhibition of PRC2 activity by a gainof-function $\mathrm{H} 3$ mutation found in pediatric glioblastoma. Science 340:857-861, 2013

23. López-Aguilar JE, Velázquez-Flores MA, Simón-Martínez LA, Ávila-Miranda R, Rodríguez-Florido MA, Ruiz-Esparza Garrido R: Circulating microRNAs as biomarkers for pediatric astrocytomas. Arch Med Res 48:323-332, 2017

24. Louis DN, Giannini C, Capper D, Paulus W, Figarella-Branger D, Lopes MB, et al: cIMPACT-NOW update 2: diagnostic clarifications for diffuse midline glioma, H3 K27M-mutant and diffuse astrocytoma/anaplastic astrocytoma, IDH-mutant. Acta Neuropathol 135:639-642, 2018

25. Louis DN, Ohgaki H, Wiestler OD, Cavenee WK: WHO Classification of Tumours of the Central Nervous System, rev ed 4. Lyon: IARC Press, 2016

26. Louis DN, Perry A, Reifenberger G, von Deimling A, Figarella-Branger D, Cavenee WK, et al: The 2016 World Health Organization Classification of Tumors of the Central Nervous System: a summary. Acta Neuropathol 131:803-820, 2016

27. MacKay A, Burford A, Molinari V, Jones DTW, Pfister SM, Wurdinger T, et al: Integrated molecular and pathological characterisation of non-brainstem paediatric high grade glioma from the HERBY phase II randomised trial. Neuro Oncol 19 (Suppl 4):iv23, 2017

28. Meissner A, Mikkelsen TS, Gu H, Wernig M, Hanna J, Sivachenko A, et al: Genome-scale DNA methylation maps of pluripotent and differentiated cells. Nature 454:766-770, 2008

29. Merchant TE, Pollack IF, Loeffler JS: Brain tumors across the age spectrum: biology, therapy, and late effects. Semin Radiat Oncol 20:58-66, 2010

30. Moher D, Liberati A, Tetzlaff J, Altman DG: Preferred reporting items for systematic reviews and meta-analyses: the PRISMA statement. PLoS Med 6:e1000097, 2009 
31. Morales La Madrid A, Hashizume R, Kieran MW: Future clinical trials in DIPG: bringing epigenetics to the clinic. Front Oncol 5:148, 2015

32. Nikbakht H, Panditharatna E, Mikael LG, Li R, Gayden T, Osmond M, et al: Spatial and temporal homogeneity of driver mutations in diffuse intrinsic pontine glioma. Nat Commun 7:11185, 2016

33. Panditharatna E, Yaeger K, Kilburn LB, Packer RJ, Nazarian $\mathrm{J}$ : Clinicopathology of diffuse intrinsic pontine glioma and its redefined genomic and epigenomic landscape. Cancer Genet 208:367-373, 2015

34. Piunti A, Hashizume R, Morgan MA, Bartom ET, Horbinski $\mathrm{CM}$, Marshall SA, et al: Therapeutic targeting of polycomb and BET bromodomain proteins in diffuse intrinsic pontine gliomas. Nat Med 23:493-500, 2017

35. Puget S, Philippe C, Bax DA, Job B, Varlet P, Junier MP, et al: Mesenchymal transition and PDGFRA amplification/mutation are key distinct oncogenic events in pediatric diffuse intrinsic pontine gliomas. PLoS One 7:e30313, 2012

36. Reynolds N, Salmon-Divon M, Dvinge H, Hynes-Allen A, Balasooriya G, Leaford D, et al: NuRD-mediated deacetylation of H3K27 facilitates recruitment of Polycomb Repressive Complex 2 to direct gene repression. EMBO J 31:593605, 2012

37. Ryall S, Krishnatry R, Arnoldo A, Buczkowicz P, Mistry M, Siddaway R, et al: Targeted detection of genetic alterations reveal the prognostic impact of H3K27M and MAPK pathway aberrations in paediatric thalamic glioma. Acta Neuropathol Commun 4:93, 2016

38. Saratsis AM, Yadavilli S, Magge S, Rood BR, Perez J, Hill DA, et al: Insights into pediatric diffuse intrinsic pontine glioma through proteomic analysis of cerebrospinal fluid. Neuro Oncol 14:547-560, 2012

39. Schardt C, Adams MB, Owens T, Keitz S, Fontelo P: Utilization of the PICO framework to improve searching PubMed for clinical questions. BMC Med Inform Decis Mak 7:16, 2007

40. Sterne JAC, Jüni P, Schulz KF, Altman DG, Bartlett C, Egger M: Statistical methods for assessing the influence of study characteristics on treatment effects in 'meta-epidemiological' research. Stat Med 21:1513-1524, 2002

41. Szenker E, Ray-Gallet D, Almouzni G: The double face of the histone variant H3.3. Cell Res 21:421-434, 2011

42. Vanan MI, Eisenstat DD: Management of high-grade gliomas in the pediatric patient: past, present, and future. Neurooncol Pract 1:145-157, 2014

43. Venneti S, Garimella MT, Sullivan LM, Martinez D, Huse JT, Heguy A, et al: Evaluation of histone 3 lysine 27 tri- methylation (H3K27me3) and enhancer of zest 2 (EZH2) in pediatric glial and glioneuronal tumors shows decreased H3K27me3 in H3F3A K27M mutant glioblastomas. Brain Pathol 23:558-564, 2013

44. Venneti S, Santi M, Felicella MM, Yarilin D, Phillips JJ, Sullivan LM, et al: A sensitive and specific histopathologic prognostic marker for H3F3A K27M mutant pediatric glioblastomas. Acta Neuropathol 128:743-753, 2014

45. Warren KE: Diffuse intrinsic pontine glioma: poised for progress. Front Oncol 2:205, 2012

46. Wells G, Shea B, O'Connell D, Peterson J, Welch V, Losos $M$, et al: The Newcastle-Ottawa Scale (NOS) for Assessing the Quality of Nonrandomised Studies in Meta-Analyses. Ottawa: Ottawa Hospital Research Institute, 2009 (http:// www.ohri.ca/programs/clinical_epidemiology/oxford.asp) [Accessed October 3, 2018]

\section{Disclosures}

The authors report no conflict of interest concerning the materials or methods used in this study or the findings specified in this paper.

\section{Author Contributions}

Conception and design: Lu, Alvi, Daniels. Acquisition of data: Lu, Alvi. Analysis and interpretation of data: Lu, Alvi. Drafting the article: all authors. Critically revising the article: all authors. Reviewed submitted version of manuscript: Lu, Alvi, Daniels. Approved the final version of the manuscript on behalf of all authors: Lu. Statistical analysis: Lu. Study supervision: Daniels.

\section{Supplemental Information Online-Only Content}

Supplemental material is available with the online version of the article.

Supplementary Data. https://thejns.org/doi/suppl/10.3171/ 2018.9.PEDS18419.

\section{Correspondence}

Victor M. Lu: Prince of Wales Clinical School, University of New South Wales, Randwick, NSW, Australia. victor.lu@student.unsw. edu.au. 